\title{
COMBINING ABILITY EFFECTS OF SOME NEW YELLOW MAIZE INBRED LINES.
}

\author{
A. A. Abd El-Mottalb \\ Maize Research Department, Field Crops Research Institute, ARC. \\ Received: Mar. 27,2017 \\ Accepted: May 27, 2017
}

\begin{abstract}
Fifteen newly developed yellow maize inbred lines were topcrossed to each of three inbred line testers, i.e. Gz. 658, Sd. 3118 and Sd. 3120 at Sids Agricultural Research Station during 2014 growing season. In 2015 season, resulting 45 topcrosses along with three commercial hybrids as check varieties, i.e. SC.162, SC.168 and SC.176, were evaluated in a replicated yield trail conducted at Gemmeiza and Sids. Data were recorded for days to 50\% silking, plant and ear height (cm.), ear length (cm), ear diameter (cm) and grain yield adjusted to $15.5 \%$ grain moisture. Combined analysis over the tow locations showed significant differences between the two locations for all studied traits except for grain yield. Mean squares due to crosses, lines and testers were significant for all studied traits. Mean squares due to lines $x$ testers were significant for plant and ear height, ear diameter and grain yield. Mean squares due to lines $x$ locations were significant for all studied traits except for ear diameter. Mean squares due to testers $x$ locations were significant for all studied traits except for ear length. Mean squares due to lines $x$ testers $x$ locations interaction were highly significant for all studied traits except for ear length and ear diameter. The magnitude of $\delta^{2}$ GCA (average) was larger than that of $\delta^{2}$ SCA for days to $50 \%$ silking, plant height and grain yield. It was recommended that inbred lines 1, 2 and 4 which possessed the highest GCA effects for grain yield may be considered promising lines for improving grain yield. Also, the crosses ( $L 7 x G z$. 658), (L8 $x$ Gz. 658) and (L10 x Sd 3118) may be released for commercial cultivation after further testing and evaluation.
\end{abstract}

Key words: Maize, Zea mays, line $x$ tester , Combining ability, Top crosses

\section{INTRODUCTION}

Increasing the cultivated area of yellow maize is one of the most important objectives of the Egyptian state, in order to limit its importation and provide foreign currency. Yellow maize is used to feed poultry and animals because corn contains protein and oil as well as carotene and vitamin $\mathrm{E}$.

Topcross testing as suggested by Davis (1927) has become a standard procedure for evaluating the general combining ability of inbred lines to be used in hybrid development. The concept of general G.C.A. and specific S.C.A. combining ability was firstly defined by Sprague and Tatum (1942). Line $x$ tester analysis provides information about general and specific combining abilities of inbred parents and is helpful in estimating various types of gene action
( Singh and Chaudhary 1985). Many investigators suggested that G.C.A. effects were relatively more important than S.C.A effects in inbred lines ( Shehata 1992, ElZeir et al. 1993, and El-Zeir 1999). While (Lonnquiest and Gardener (1961) and Shehata and Dhawan (1975) found that the S.C.A. effects were more important than G.C.A. effects in the inheritance of grain yield. The main objectives of this study were:

(1) to identify the best inbred lines for general combining ability,

(2) to mark the best crosses regarding the specific combining ability for grain yield and other traits and

(3) to determine the different types of gene action involved in the manifestation of grain yield and other studied traits. 


\section{MATERIALS AND METHODS}

Fifteen new yellow maize inbred lines developed at Sids Agricultural Research Station were used in this study. In 2014 growing season the fifteen inbred lines were topcrossed to each of three narrow base inbred line testers; i.e Gz. 658 , Sd.3118 and Sd.3120 at Sids Agricultural Research Station. In 2015 season the 45 crosses along with three commercial hybrids as check varieties; i.e. SC.162, SC.168 and SC.176; were evaluated in a yield trail conducted at Gemmeiza and Sids Agric. Res. Stns. A randomized Complete block design (RCBD) with four replications was used in both location. The experimental plot was one row, $6.0 \mathrm{~m}$ long and $0.80 \mathrm{~m}$ wide. Sowing was made in hills evenly spaced at $0.25 \mathrm{~m}$ along the row. All cultural practices for maize cultivation were performed as recommended. Data were recorded for number of days to $50 \%$ silking, plant and ear height $(\mathrm{cm}$.$) , ear length (\mathrm{cm})$, ear diameter $(\mathrm{cm})$ and grain yield in ardab faddan ${ }^{-1}$. Grain yield was adjusted to $15.5 \%$ moisture content and was converted into ardab per faddan ( one $a r d a b=140 \mathrm{Kg}$ ). Analysis of variance was performed for the combined data over two locations according to Steel and Torrie (1980). Bartlett test was used to test the homogeneity of error variance among locations for all studied traits. Procedures of Kempthorne (1957) were performed to obtain valuable information about the combining ability of lines and testers.

\section{RESULTS AND DISCUSSION Analysis of variance}

Combined analyses of variance over two locations of the 45 topcrosses for the studied traits are presented in Table (1). Mean squares of locations were highly significant for all studied traits indicatig differences between the two locations except for grain yield where the differences were not significant. Mean squares due to crosses and lines were highly significant for all the studied traits indicating that significant differences exist among both lines and crosses. Mean squares due to testers were either significant or highly significant for all studied traits indicating that testers were different. Mean squares due to lines $x$ testers were highly significant for plant and ear height, ear diameter and grain yield indicating that lines differed in their order of performance when crossed with each of the testers for these traits. Similar results were obtained by Soliman and Sadek (1999), Soliman (2000), Amer et al. (2003), Abd ElAzeem et al. (2004), Abd El-Moula and Abd El-Aal (2009), Abd El-Azeem et al. (2010), Abd El-mottalb (2015) and Moshera et al. (2016). Mean squares for the interactions of crosses, lines and testers, as well as those of lines $x$ testers $x$ locations, were highly significant for all studied traits, except for the trait of ear length which was highly significant for only the interaction of lines $x$ locations, and for the trait of ear diameter which was significant only for the interaction of testers $x$ locations. These significant of interactions indicate that the crosses, lines, and testers performed differently from location to another. These results are in agreement with Mahmoud and Abd ElAzeem (2004), Abd El-Mola and Abd El-Aal (2009), Abd El-Azeem et al. (2010), Ibrahim et al. (2010) and Abd El-Azeem (2011). They found that the interaction of lines $x$ testers $x$ locations was highly significant for grain yield. The magnitude of mean squares due to testers was higher than that due to lines for all studied traits except for ear length indicating that the tester contributed much more to the total variation for most of the studied traits. Also, mean squares due to testers $x$ locations were higher than those of lines $x$ locations for all studied traits indicating that the testers were more affected by the environmental conditions than the lines. These results are in agreement with those obtained by ElMorshidy et al. (2003), Abd El-Moula and ElAal (2009), Ibrahim et al. (2010), Abd ElAzeem (2011), Abd El-mottalb (2014) and Abd El-mottalb (2015). 
Combining ability effects of some new yellow maize inbred lines.

Table 1. Mean squares of the combined performance across two locations for the studied traits (2015 season).

\begin{tabular}{|c|c|c|c|c|c|c|c|}
\hline \multirow[b]{2}{*}{ SO V } & \multirow[b]{2}{*}{$d f$} & \multicolumn{6}{|c|}{ Mean Squares } \\
\hline & & $\begin{array}{l}\text { Days to } \\
50 \% \text { silking }\end{array}$ & $\begin{array}{l}\text { Plant } \\
\text { height } \\
(\mathrm{cm})\end{array}$ & $\begin{array}{l}\text { Ear height } \\
\quad(\mathrm{cm})\end{array}$ & $\begin{array}{l}\text { Ear length } \\
\quad(\mathrm{cm})\end{array}$ & $\begin{array}{c}\text { Ear } \\
\text { diameter } \\
(\mathrm{cm})\end{array}$ & $\begin{array}{l}\text { Grain yield } \\
\left(\text { ard fad }^{-1}\right)\end{array}$ \\
\hline Location (E) & 1 & $2662.34^{\star *}$ & $17710.07^{* *}$ & $36703.40^{* *}$ & $245.03^{* *}$ & $23.61^{* *}$ & 12.17 \\
\hline Rep/Loc & 6 & 5.70 & 285.72 & 276.92 & 2.09 & 0.157 & 16.50 \\
\hline Crosses (C) & 44 & $6.100^{* *}$ & $1741.70^{* *}$ & $512.84^{* *}$ & $4.27^{* *}$ & $0.089^{* *}$ & $39.02^{* *}$ \\
\hline Lines ( L ) & 14 & $7.30^{* *}$ & $559.79^{* *}$ & $455.68^{\star *}$ & $8.95^{\star *}$ & $0.141^{* *}$ & $49.92^{\star *}$ \\
\hline Testers ( $\mathrm{T}$ ) & 2 & $66.29^{\star \star}$ & $29443.96^{* *}$ & $5300.21^{\star *}$ & $5.49^{*}$ & $0.169^{\star *}$ & $235.54^{\star *}$ \\
\hline $\mathrm{L} \times \mathrm{T}$ & 28 & 1.20 & $353.93^{* *}$ & $199.46^{* *}$ & 1.85 & $0.057^{* *}$ & $19.54^{* *}$ \\
\hline C $\times$ LOC & 44 & $5.21^{\star *}$ & $1101.55^{\star \star}$ & $373.29^{\star *}$ & 2.18 & 0.040 & $27.23^{* *}$ \\
\hline L $\times$ Loc & 14 & $4.51^{\star \star}$ & $297.87^{\star *}$ & $264.41^{\star *}$ & $3.85^{\star \star}$ & 0.047 & $25.19^{\star *}$ \\
\hline TX LOC & 2 & $44.80^{* *}$ & $19450.07^{* *}$ & $4401.32^{* \star}$ & 3.88 & $0.122^{*}$ & $148.64^{\star *}$ \\
\hline $\mathrm{L} \times \mathrm{T} \times \mathrm{LOC}$ & 28 & $2.73^{\star *}$ & $192.78^{\star *}$ & $140.01^{* *}$ & 1.22 & 0.030 & $19.58^{\star *}$ \\
\hline Pooled error & $\begin{array}{c}26 \\
4\end{array}$ & 1.27 & 101.01 & 75.93 & 1.48 & 0.030 & 8.38 \\
\hline C V\% & & 1.86 & 4.19 & 6.29 & 6.57 & 6.92 & 10.86 \\
\hline
\end{tabular}

\section{Mean performance}

Mean performance of the 45 crosses for all studied traits is presented in Tables (2) and (3) . For days to $50 \%$ silking, the earliest cross was L4 x Sd. 3118 . Plant height ranged from 200.00 for cross $L 8 \times$ Gz. 658 to 255.63 for cross L12 x Sd. 3118 . Only one cross, i.e L8 x Gz 658, was significantly shorter than the shortest check hybrid SC.168. The same cross ( $\mathrm{L} 8 \times \mathrm{Gz}$ 658 ) possessed the lowest ear placement among tested crosses and was significantly lower than the best performing check hybrid (SC.168) regarding ear height. For ear length, non of the crosses possessed longer ears than those of the best performing check hybrid (SC.162). For ear diameter, all crosses exhibited ear diameter values similar to those of the best performing check hybrid (SC 168) since no significant differences. For grain yield, fifteen crosses, i.e L1 x Sd. 3118, L1 x Sd. 3120, L2 x Sd. 3118, L2 x Sd. 3120, L3 x Sd. 3120, L4 x Gz. 658, L4 x Sd. 3118, L4 x Sd. 3120, L9 x Gz. 658, L9 x Sd. 3120, L10 x Sd. 3118, L12 x Sd. 3120, L13 x Sd. 3120, L15 x Sd. 3118 and L15 x Sd. 3120, significantly outyielded the highest yielding check hybrid SC.176 .

General combining ability effects:
General combining ability effects are presented in Table (4) . For days to $50 \%$ silking, two inbred lines, i.e. L4 and L15, exhibited highly significant and significant negative GCA effects, respectively. These inbred lines are considered the best inbred lines when breeding for earliness. Concerning plant height the four inbred lines L $3, L$ 7, L8 and L 15 manifested significant and highly significant negative GCA effects. Regarding ear height, inbred lines L 7 , L8 and L15 possessed significant and highly significant negative GCA effects. For ear length the inbred lines L 1, L8, L12 and L15 possessed significant and highly significant positive GCA effects. Regarding the inbred line testers, the data showed the tester $\mathrm{Gz}$. 658 to posses more favorable effects than inbred line testers Sd. 3118 and Sd. 3120. For ear diameter, inbred lines L 2, L4 and L12 exhibited significant and highly significant positive GCA effects. For grain yield three inbred lines, i.e L1, L2 and L4 showed significant and highly significant positive GCA effects indicating that they have favorable genes for productivity and could be good combiners for grain yield. The tester inbred line Sd. 3120 had highly significant positive GCA effects and could be considered as a good combiner for grain yield. 
Table 2. Mean performance of the crosses and check hybrids combined over locations for days to $\mathbf{5 0 \%}$ silking, plant height, and ear height, 2015 season.

\begin{tabular}{|c|c|c|c|c|c|c|c|c|c|}
\hline \multirow{2}{*}{ Lines } & \multicolumn{3}{|c|}{ Days to $50 \%$ silking } & \multicolumn{3}{|c|}{ Plant height $(\mathrm{cm})$} & \multicolumn{3}{|c|}{ Ear height $(\mathrm{cm})$} \\
\hline & Gz.658 & Sd.3118 & Sd.3120 & Gz.658 & Sd.3118 & Sd.3120 & Gz.658 & Sd.3118 & Sd.3120 \\
\hline L1 & 62.00 & 60.50 & 59.75 & 232.50 & 243.75 & 242.50 & 140.00 & 143.75 & 142.50 \\
\hline L2 & 61.25 & 59.63 & 60.00 & 230.63 & 246.88 & 238.13 & 141.88 & 140.00 & 133.75 \\
\hline L3 & 62.75 & 61.38 & 61.00 & 209.38 & 243.13 & 241.25 & 123.13 & 148.13 & 146.88 \\
\hline L4 & 60.50 & 58.88 & 59.25 & 220.00 & 248.75 & 247.50 & 130.63 & 142.50 & 143.75 \\
\hline L5 & 61.38 & 59.75 & 59.75 & 215.00 & 250.00 & 250.00 & 125.63 & 148.75 & 140.63 \\
\hline L6 & 61.25 & 60.13 & 59.25 & 216.25 & 250.00 & 252.50 & 126.25 & 147.50 & 146.25 \\
\hline L7 & 61.25 & 61.13 & 60.50 & 213.13 & 235.00 & 238.13 & 123.75 & 133.75 & 133.13 \\
\hline L8 & 61.63 & 59.88 & 60.38 & 200.00 & 234.38 & 250.00 & 116.25 & 133.13 & 138.13 \\
\hline L9 & 61.38 & 59.88 & 60.25 & 218.13 & 248.13 & 240.63 & 129.38 & 145.00 & 136.25 \\
\hline L10 & 62.63 & 61.38 & 60.63 & 229.38 & 252.50 & 245.00 & 140.63 & 149.38 & 149.38 \\
\hline L11 & 60.75 & 60.50 & 59.75 & 216.88 & 249.38 & 240.00 & 131.25 & 140.00 & 138.13 \\
\hline L12 & 60.75 & 59.75 & 60.00 & 216.25 & 255.63 & 253.75 & 132.50 & 143.75 & 142.50 \\
\hline L13 & 61.63 & 59.88 & 60.13 & 215.63 & 247.50 & 254.38 & 126.87 & 136.25 & 141.25 \\
\hline L14 & 60.75 & 60.63 & 60.25 & 213.75 & 243.13 & 243.13 & 130.00 & 143.13 & 142.50 \\
\hline L15 & 61.00 & 59.25 & 59.88 & 221.25 & 231.88 & 233.13 & 130.00 & 130.00 & 141.25 \\
\hline $\begin{array}{l}\text { Checks } \\
\text { SC.162 } \\
\text { SC.168 } \\
\text { SC. } 176\end{array}$ & \multicolumn{3}{|c|}{$\begin{array}{l}63.63 \\
61.63 \\
60.13 \\
\end{array}$} & \multicolumn{3}{|c|}{$\begin{array}{l}271.25 \\
210.63 \\
263.75 \\
\end{array}$} & \multicolumn{3}{|c|}{$\begin{array}{l}153.63 \\
126.13 \\
135.75\end{array}$} \\
\hline LSD $_{0.05}$ & \multicolumn{3}{|c|}{1.11} & \multicolumn{3}{|c|}{9.73} & \multicolumn{3}{|c|}{4.44} \\
\hline
\end{tabular}

Table 3. Mean performance of the crosses and check hybrids at the combined over locations for ear length, ear diameter, and grain yield, 2015 season.

\begin{tabular}{|c|c|c|c|c|c|c|c|c|c|}
\hline \multirow{2}{*}{ Lines } & \multicolumn{3}{|c|}{ Ear length $(\mathrm{cm})$} & \multicolumn{3}{|c|}{ Ear diameter $(\mathrm{cm})$} & \multicolumn{3}{|c|}{ Grain yield } \\
\hline & Gz.658 & Sd.3118 & Sd.3120 & Gz.658 & Sd.3118 & Sd.3120 & Gz.658 & Sd.3118 & Sd.3120 \\
\hline L1 & 20.33 & 19.88 & 19.80 & 4.73 & 4.75 & 4.75 & 24.78 & 28.06 & 30.19 \\
\hline L2 & 19.75 & 20.25 & 19.25 & 4.85 & 4.93 & 5.05 & 25.23 & 27.54 & 30.78 \\
\hline L3 & 17.70 & 17.68 & 18.48 & 4.70 & 4.85 & 4.85 & 25.58 & 26.26 & 28.77 \\
\hline L4 & 19.35 & 18.33 & 18.48 & 4.83 & 5.00 & 5.13 & 28.06 & 30.36 & 29.60 \\
\hline L5 & 18.93 & 19.15 & 18.65 & 4.75 & 5.03 & 4.83 & 21.64 & 25.82 & 23.47 \\
\hline L6 & 19.13 & 18.43 & 18.35 & 4.80 & 4.88 & 4.80 & 25.96 & 25.71 & 25.11 \\
\hline L7 & 19.88 & 18.48 & 20.00 & 4.80 & 4.70 & 4.80 & 25.53 & 23.09 & 26.30 \\
\hline L8 & 19.93 & 19.58 & 20.20 & 4.80 & 4.65 & 4.85 & 26.08 & 23.36 & 27.11 \\
\hline L9 & 18.98 & 19.33 & 19.60 & 4.80 & 4.73 & 4.60 & 27.25 & 26.82 & 27.24 \\
\hline L10 & 19.60 & 19.45 & 19.58 & 4.73 & 4.80 & 4.78 & 24.86 & 29.14 & 26.21 \\
\hline L11 & 19.98 & 19.75 & 19.53 & 4.83 & 4.80 & 4.80 & 24.03 & 26.62 & 26.68 \\
\hline L12 & 20.05 & 20.75 & 19.45 & 4.80 & 5.00 & 4.93 & 23.29 & 25.55 & 28.30 \\
\hline L13 & 19.55 & 18.85 & 19.38 & 4.75 & 4.90 & 4.93 & 24.34 & 26.98 & 29.06 \\
\hline L14 & 20.53 & 18.78 & 19.63 & 4.83 & 4.78 & 4.98 & 20.88 & 26.53 & 26.46 \\
\hline L15 & 20.60 & 19.20 & 20.28 & 4.78 & 4.85 & 4.75 & 25.29 & 27.40 & 29.05 \\
\hline Cheeks & \multirow{4}{*}{\multicolumn{3}{|c|}{$\begin{array}{l}22.28 \\
20.53 \\
20.60\end{array}$}} & \multirow{4}{*}{\multicolumn{3}{|c|}{4.78}} & \multirow{4}{*}{\multicolumn{3}{|c|}{$\begin{array}{l}20.72 \\
23.85 \\
24.34\end{array}$}} \\
\hline SC.162 & & & & & & & & & \\
\hline SC.168 & & & & & & & & & \\
\hline SC.176 & & & & & & & & & \\
\hline $\operatorname{LSD}_{0.05}$ & \multicolumn{3}{|c|}{1.26} & \multicolumn{3}{|c|}{0.33} & \multicolumn{3}{|c|}{2.78} \\
\hline
\end{tabular}


Table 4 . General combining ability effects $\left(\hat{g}_{i}\right)$ for the tested lines and testers ( combined across two locations, 2015 season).

\begin{tabular}{|c|c|c|c|c|c|c|}
\hline Lines & $\begin{array}{l}\text { Days to } \\
50 \% \text { silking }\end{array}$ & $\begin{array}{c}\text { Plant height } \\
\text { (cm) }\end{array}$ & $\begin{array}{l}\text { Ear height } \\
\quad(\mathrm{cm})\end{array}$ & $\begin{array}{l}\text { Ear length } \\
\quad(\mathrm{cm})\end{array}$ & $\begin{array}{c}\text { Ear diameter } \\
(\mathrm{cm})\end{array}$ & $\begin{array}{l}\text { Grain yield } \\
\left(\operatorname{ard~fad~}^{-1}\right)\end{array}$ \\
\hline L1 & 0.214 & 3.625 & $4.542^{* *}$ & $0.606^{\star *}$ & -0.084 & $1.312^{*}$ \\
\hline L2 & -0.244 & 2.583 & 1.00 & 0.356 & $0.116^{\star *}$ & $1.486^{*}$ \\
\hline L3 & $1.172^{* *}$ & $-4.708^{\star}$ & 1.833 & $-1.444^{* *}$ & -0.026 & 0.505 \\
\hline L4 & $-0.994^{* *}$ & 2.792 & 1.417 & $-0.677^{* *}$ & $0.157^{* *}$ & $2.977^{* *}$ \\
\hline L5 & -0.244 & 2.375 & 0.792 & $-0.486^{*}$ & 0.041 & $-2.720^{* *}$ \\
\hline L6 & -0.328 & 3.625 & 2.458 & $-0.761^{* *}$ & -0.001 & -0.771 \\
\hline L7 & 0.422 & $-7.208^{* *}$ & $-7.333^{* *}$ & 0.056 & -0.059 & $-1.391^{*}$ \\
\hline L8 & 0.089 & $-7.833^{\star *}$ & $-8.375^{\star \star}$ & $0.506^{*}$ & -0.059 & -0.846 \\
\hline L9 & -0.036 & -0.333 & -0.667 & -0.094 & $-0.118^{* *}$ & 0.738 \\
\hline L10 & $1.006^{* *}$ & $6.333^{* *}$ & $8.917^{* \star}$ & 0.148 & -0.059 & 0.371 \\
\hline L11 & -0.203 & -0.542 & -1.083 & 0.356 & -0.018 & -0.586 \\
\hline L12 & -0.369 & $5.917^{\star}$ & 2.042 & $0.689^{* *}$ & $0.082^{*}$ & -0.649 \\
\hline L13 & 0.006 & 3.208 & -2.75 & -0.136 & 0.032 & 0.431 \\
\hline L14 & 0.006 & -2.625 & 1.00 & 0.248 & 0.032 & $-1.738^{* *}$ \\
\hline L15 & $-0.494^{*}$ & $-7.208^{* *}$ & $-3.792^{*}$ & $0.631^{* *}$ & -0.034 & 0.881 \\
\hline SE gi & 0.230 & 2.051 & 1.778 & 0.248 & 0.035 & 0.590 \\
\hline $\begin{array}{l}\text { Tester } \\
\text { Gz.658 }\end{array}$ & $0.856^{* *}$ & $-18.083^{\star *}$ & $-7.667^{* *}$ & $0.223^{*}$ & $-0.043^{* *}$ & $-1.510^{\star *}$ \\
\hline Sd.3118 & $-0.369 * *$ & $9.375^{\star *}$ & $4.125^{\star \star}$ & -0.204 & 0.016 & 0.252 \\
\hline Sd.3120 & $-0.486^{\star *}$ & $8.708^{\star *}$ & $3.542^{* *}$ & -0.019 & 0.027 & $1.258^{\star \star}$ \\
\hline SE gi & 0.102 & 0.917 & 0.795 & 0.111 & 0.015 & 0.264 \\
\hline
\end{tabular}

${ }^{*},{ }^{* *}$ Indicate significance at 0.05 and 0.01 levels of probability, respectively.

Specific combining ability effects:-

Specific combining ability effects of the 45 topcrosses for all studied traits are given in Tables (5) and (6). Results showed that non of the crosses exhibited desirable SCA effects ( negative effects ) for days to $50 \%$ silking. For plant height, three crosses, i.e (L2 x Sd. 3120), (L8 x Gz. 658) and (L12 x Gz. 658) exhibited significant and highly significant negative SCA effects (desirable effects ). For ear height, four crosses, i.e (L2 x Sd. 3120), (L3 x Gz. 658), (L6 x Gz.
658) and (L15 x Sd. 3118) possessed significant and highly significant negative SCA effects. One cross, i.e ( L12 x Sd. 3118) had significant positive SCA effects for ear length. Similarly, one cross, i.e ( $L 5 \mathrm{X}$ Sd. 3118 ) showed significant positive SCA effects for ear diameter. For grain yield three crosses, i.e ( L7 x Gz. 658), (L8 x Gz. 658) and (L10 $\times$ Sd. 3118) had significant positive SCA effects with values $2.071,2.073$ and 2.150 respectively . 


\section{Variance Components :-}

Estimates of combining ability variances and their interactions with locations are presented in Table (7). The results showed that $\delta^{2} \mathrm{GCA}-\mathrm{L}$ was higher than $\delta^{2} \mathrm{GCA}-\mathrm{T}$ for ear length, ear diameter and grain yield, indicating that most of GCA variance was due to lines for these traits, while $\delta^{2} \mathrm{GCA}-\mathrm{T}$ was higher than $\delta^{2}$ GCA- $L$ for days to $50 \%$ silking, plant height and ear height, indicating that most of GCA variance was due to testers for such traits. The magnitude of $\sigma 2$ GCA average was larger than that obtained for $\sigma 2$ SCA for days to $50 \%$ silking, plant height, and grain yield, indicating that the additive type of gene action played an important role in the inheritance of these traits. While $\sigma 2 \mathrm{SCA}$ was larger than that obtained for $\sigma 2$ GCA for ear height, ear length, and ear diameter indicating that the non-additive type of gene action was more important in the inheritance of these traits. More over, the magnitude of o2 GCA x loc. interaction was higher than o2 SCA $x$ loc. for days to $50 \%$ silking, plant and ear height, ear length, and ear diameter indicating that the additive type of gene action was more affected by the environmental conditions than non-additive ones. These results are in agreement with those obtained by Soliman and Sadek (1999), Abd El-Azeem (2011) and Abd ElMottalb et al. (2013). On the other hand, $\sigma 2$ SCA $x$ loc. interaction was higher than $\sigma 2$ GCA $\times$ loc. for grain yield, indicating that non-additive gene action was more biased by the interaction with the environment than the additive effects. This result is in agreement with those reported by Sedhom (1992), Al-Naggar et al. (1997), El-Itriby et al. (1990), El-Zeir et al. (1993), Abd ElAzeem (2000) and Abd El-Mottalb, (2014).

Table 5. Estimated specific combining ability effects of 45 crosses for days to 50\%silking, plant height and ear height ( combined across two locations, 2015 season).

\begin{tabular}{|c|c|c|c|c|c|c|c|c|c|}
\hline \multirow{2}{*}{ Lines } & \multicolumn{3}{|c|}{ Days to $50 \%$ silking } & \multicolumn{3}{|c|}{ Plant height $(\mathrm{cm})$} & \multicolumn{3}{|c|}{ Ear height (cm) } \\
\hline & Gz.658 & Sd.3118 & Sd.3120 & Gz.658 & Sd.3118 & Sd.3120 & Gz.658 & Sd.3118 & Sd.3120 \\
\hline L1 & 0.394 & 0.119 & -0.514 & $11.00^{* *}$ & -5.208 & -5.792 & 5.583 & -2.458 & -3.125 \\
\hline L2 & 0.103 & -0.297 & 0.194 & $10.167^{\star \star}$ & -1.042 & $-9.125^{\star}$ & $11.00^{* *}$ & -2.667 & $-8.333^{* *}$ \\
\hline L3 & 0.186 & 0.036 & -0.222 & -3.792 & 2.50 & 1.292 & $-8.583^{\star *}$ & 4.625 & 3.958 \\
\hline L4 & 0.103 & -0.297 & 0.194 & -0.667 & 0.625 & 0.042 & -0.667 & -0.583 & 1.25 \\
\hline L5 & 0.228 & -0.172 & -0.056 & -5.250 & 2.292 & 2.958 & -5.042 & $6.292^{*}$ & -1.25 \\
\hline L6 & 0.186 & 0.286 & -0.432 & -5.250 & 1.042 & 4.208 & $-6.083^{*}$ & 3.375 & 2.708 \\
\hline L7 & -0.564 & 0.536 & 0.028 & 2.458 & -3.125 & 0.667 & 1.208 & -0.583 & -0.625 \\
\hline L8 & 0.144 & -0.381 & 0.236 & $-10.042^{* *}$ & -3.125 & $13.167^{* *}$ & -5.25 & -0.167 & 5.417 \\
\hline L9 & 0.019 & -0.256 & 0.236 & 0.583 & 3.125 & -3.708 & 0.167 & 4.00 & -4.167 \\
\hline L10 & 0.228 & 0.203 & -0.431 & 5.167 & 0.833 & -6.00 & 1.833 & -1.208 & -0.625 \\
\hline L11 & -0.439 & 0.536 & -0.097 & -0.458 & 4.583 & -4.125 & 2.458 & -0.583 & -1.875 \\
\hline L12 & -0.272 & -0.047 & 0.319 & $-7.542^{*}$ & 4.375 & 3.167 & 0.583 & 0.042 & -0.625 \\
\hline L13 & 0.228 & -0.297 & 0.069 & -5.458 & -1.042 & 6.5 & -0.25 & -2.667 & 2.917 \\
\hline L14 & -0.647 & 0.453 & 0.194 & -1.50 & 0.417 & 1.083 & -0.875 & 0.458 & 0.417 \\
\hline L15 & 0.103 & -0.422 & 0.319 & $10.583^{\star *}$ & -6.25 & -4.333 & 3.917 & $-7.875^{\star *}$ & 3.958 \\
\hline SE Sij & & 0.398 & & & 3.553 & & & 3.080 & \\
\hline
\end{tabular}


Table 6. Estimated specific combining ability effects of 45 crosses for ear length, ear diameter and grain yield ( combined across two locations, 2015 season).

\begin{tabular}{|c|c|c|c|c|c|c|c|c|c|}
\hline \multirow{2}{*}{ Lines } & \multicolumn{3}{|c}{ Ear length (cm) } & \multicolumn{3}{c|}{ Ear diameter (cm) } & \multicolumn{3}{c|}{ Grain yield $\left(\mathrm{ard} \mathrm{fad}^{-1}\right)$} \\
\cline { 2 - 11 } & Gz.658 & Sd.3118 & Sd.3120 & Gz.658 & Sd.3118 & Sd.3120 & Gz.658 & Sd.3118 & Sd.3120 \\
\hline L1 & 0.102 & 0.079 & -0.181 & 0.026 & -0.007 & -0.019 & -1.391 & 0.133 & 1.258 \\
\hline L2 & -0.223 & 0.704 & -0.481 & -0.049 & -0.032 & 0.081 & -1.111 & -0.561 & 1.671 \\
\hline L3 & -0.473 & -0.071 & 0.544 & -0.057 & 0.034 & 0.022 & 0.222 & -0.862 & 0.640 \\
\hline L4 & 0.411 & -0.188 & -0.223 & -0.116 & 0.001 & 0.114 & 0.230 & 0.770 & -0.999 \\
\hline L5 & -0.206 & 0.446 & -0.239 & -0.074 & $0.143^{*}$ & -0.069 & -0.491 & 1.925 & -1.434 \\
\hline L6 & 0.269 & -0.004 & -0.264 & 0.018 & 0.034 & -0.052 & 1.879 & -0.139 & -1.739 \\
\hline L7 & 0.202 & -0.771 & 0.569 & 0.076 & -0.082 & 0.006 & $2.071^{*}$ & -2.139 & 0.067 \\
\hline L8 & -0.198 & -0.121 & 0.319 & 0.076 & $-0.132^{*}$ & 0.056 & $2.073^{*}$ & $-2.406^{*}$ & 0.333 \\
\hline L9 & 0.548 & 0.229 & 0.319 & 0.134 & 0.001 & $-0.136^{*}$ & 1.656 & -0.534 & -1.123 \\
\hline L10 & -0.164 & 0.112 & 0.052 & 0.001 & 0.018 & -0.019 & -0.368 & $2.150^{*}$ & -1.782 \\
\hline L11 & 0.002 & 0.204 & -0.206 & 0.059 & -0.023 & -0.036 & -0.235 & 0.592 & -0.358 \\
\hline L12 & -0.256 & $0.871^{*}$ & -0.614 & -0.066 & 0.076 & -0.011 & -0.915 & -0.417 & 1.332 \\
\hline L13 & 0.069 & -0.204 & 0.136 & -0.066 & 0.026 & 0.039 & -0.941 & -0.067 & 1.008 \\
\hline L14 & 0.661 & -0.663 & 0.002 & 0.009 & -0.099 & 0.089 & $-2.232^{*}$ & 1.652 & 0.580 \\
\hline L15 & 0.352 & -0.621 & 0.269 & 0.026 & 0.043 & -0.069 & -0.448 & -0.098 & 0.546 \\
\hline SE Sij & & 0.430 & & & 0.061 & & & 1.023 & \\
\hline
\end{tabular}

${ }^{*}$ Indicate significance at 0.05 level of probability

Table 7. Estimates of combining ability variances and their interactions with locations for six traits, (combined across two locations, 2015 season).

\begin{tabular}{|c|c|c|c|c|c|c|}
\hline Parameter & $\begin{array}{c}\text { Days to } \\
50 \% \\
\text { silking }\end{array}$ & $\begin{array}{l}\text { Plant } \\
\text { height } \\
(\mathrm{cm})\end{array}$ & $\begin{array}{l}\text { Ear height } \\
\quad(\mathrm{cm})\end{array}$ & $\begin{array}{l}\text { Ear length } \\
\quad(\mathrm{cm})\end{array}$ & $\begin{array}{c}\text { Ear } \\
\text { diameter } \\
(\mathrm{cm})\end{array}$ & $\begin{array}{l}\text { Grain yield } \\
\left(\text { ard fed }^{-1}\right)\end{array}$ \\
\hline$\delta^{2}$ GCA-L & 0.180 & 4.199 & 5.493 & 0.186 & 0.003 & 1.032 \\
\hline$\delta^{2}$ GCA-T & 0.192 & 81.940 & 6.995 & 0.008 & 0.000 & 0.725 \\
\hline$\delta^{2}$ GCA(average) & 0.190 & 68.983 & 6.745 & 0.038 & 0.001 & 0.776 \\
\hline$\delta^{2}$ SCA & -0.191 & 20.144 & 7.431 & 0.079 & 0.003 & -0.005 \\
\hline$\delta^{2}$ GCAL $\times$ Loc & 0.148 & 8.758 & 10.367 & 0.219 & 0.001 & 0.468 \\
\hline$\delta^{2}$ GCAT x Loc & 0.701 & 320.955 & 71.022 & 0.044 & 0.002 & 2.151 \\
\hline$\delta^{2}$ GCA x Loc & 0.609 & 268.922 & 60.913 & 0.073 & 0.002 & 1.870 \\
\hline$\delta^{2} S C A \times \operatorname{Loc}$ & 0.415 & 34.383 & 21.810 & 0.025 & -0.002 & 3.678 \\
\hline
\end{tabular}

All negative estimates of variance were considered zero 


\section{Conclusion}

Inbred lines 1, 2 and 4 which possessed the best GCA effects for grain yield may be considered promising lines for improving grain yield. Meanwhile inbred line L15 which possessed the best GCA effects for days to $50 \%$ silking, plant and ear height and ear length is recommended for developing varieties characterized with earlier maturity, shorter plants and lower ear placement. More over, the crosses ( L7 x Gz. 658), (L8 $x$ Gz. 658) and (L10 x Sd. 3118 ) may be released for commercial cultivation after further testing and evaluation.

\section{REFERENCES}

Abd El-Azeem, M.E.M. (2000). Combining ability analysis of grain yield and its components in maize. Ph.D Thesis,Fac. Agric., Moshtohor, Zagazig Univ., Egypt

Abd EL-Azeem, M.E.M, A.A. Mahmoud and A. A.M. Atia (2004). Combining ability analysis of yellow maize inbred lines. Egypt. J. Plant Breed. (8): 239-254.

Abd El-Azeem, M.E.M., M. A. Abd El-Moula and A.E.M.K. El-Galfy (2010). Estimates of combining ability for grain yield and other attributes in maize. Minufiya j. Agric. Res. Vol. 35 No.3: 927-940

Abd El-Azeem, M.E.M. (2011). Evaluation of some new white maize inbred lines using line $x$ tester analysis. Egypt. J. Plant Breed. 15(5): 99-112.

Abd El-Mottalb, A.A., M.A. Mostafa and H.Al.A. Gamea (2013). Combining ability estimates in some white maize inbred lines for yield and other traits. Egypt. J. plant Breed. 17 (3) : $13-22$.

Abd El-Mottalb, A.A. (2014). Evaluation of some yellow inbred lines for ombining ability by using topcrosses. Egypt. J. of Appl. Sci., 29 (3) 79-89.

Abd El-Mottalb, A.A. (2015). Estimates of combining ability for some new white maize inbred lines by line $x$ tester analysis. Egypt. J. plant Breed. 19 (7) : 1981 - 1992.

Abd El-Moula, M. A. and A. M. M. Abd ElAal (2009). Evaluation of some new yellow maize inbred lines via top cross analysis. Egypt. J. Appl. Sci. 24 : 148166

Al-Naggar, A.M., H.Y. El-Sherbieny and A.A. Mahmoud. (1997). Effectiveness of inbred, single crosses and population as testers for combining ability in maize. Egypt. J. Plant Breed. 1 : 35-46 .

Amer, E. A., A. A. El-Shenawy and A. A. Motawei (2003). Combining ability of new maize inbred lines via line $x$ tester analysis. Egypt. J. Plant Breed. 7 (1): 229-239.

Davis, R.L. (1927). Report of the plant breeding. Ann. Rep., Puerto Rico Agric. Exp. Stat., P: 14-15.

El-Itriby, H. A., H.Y. El-Sherbieny, M.M. Ragheb and M.A.K. Shalaby (1990). Estimation of combining ability of maize inbred lines in top crosses and its interaction with environments. Egypt. J. Appl. Sci. 5 : 354-370

El-Morshidy, M.A., E.A. Hassaballa, Sh.F. Abou-Elsaad and M.A. Abd El-Moula (2003). Combining ability and type of gene action in maize under favorable and water strees environments. Proceed. PI. Breed. Conf., Egypt. April 26, 2003: 5557.

El-Zeir, F.A.A. (1999). Evaluation of some new inbred lines for combining ability using top crosses in maize (Zea mays L). Minufiya Agric. Res. 24(5):1609-1620.

El-Zeir, F.A.A., A.A. Abdel Aziz and A.A. Galal (1993). Estimation of heterosis and combining ability effects in some new top-crosses of maize. . Minufiya, J. Agric. Res18 : 2179-2190

Ibrahim, Kh.A.M., M.A. Abd El-Moula and M.E.M. Abd El-Azeem (2010). Combining ability analysis of some yellow maize (Zea mays L.) inbred lines. Egypt. J. Agric. Res., 88(1): 33-50.

Kempthorne, O. (1957). An introduction to Genetic Statistics. John Wiley and Sons Inc., NY, USA.

Lonnquiest, J.H. and C.O. Gardner (1961). Heterosis in intervarietal crosses in 
maize and its implictions in breeding procedure. Crop Sci. 1: 179-183

Mahmoud, A. A. and M. E. Abd El-Azeem (2004). Estimates of general and specific combining ability of some yellow maize inbred lines using top-crosses. Annals of Agric. Sci., Moshtohor, Zagazig Univ., 42(2): 427-437.

Moshera, S.E. Sadek, A.A. Abd El-Mottalb and H.A.A. Gamea (2016). Estimation of combining ability for some promising white maize inbred lines through line $X$ tester mating design under different locations. Egypt. J. plant Breed. 20 (4) : $175-191$.

Sedhom, S.A. (1992). Development and evaluation of some new inbred lines of maize. Proc. $5^{\text {th }}$ Conf. Agron. Zagazig, 13-15 Sept. $1: 269-280$

Shehata, A.H. and N.L. Dhawan (1975). Genetic analysis of grain yield in maize as manifested in genetically diverse varietal populations and their crosses. Egypt. J. Genet. Cytol. 4:96-116.
Shehata, A.H. (1992). Breeding and genetic studies of maize. Ph.D.Thesis, Agron. Dep., Fac. of Agric. Menofiya Univ., Egypt.

Singh, R.K. and B.D. Chaudhary (1985). Biometrical Methods in Quantitative Genetic Analysis. Kalyani Publishers. New Delhi, 3rd Ed., P.39-68.

Soliman, F.H.S. (2000). Comparative combining ability of newly developed inbred lines of yellow maize (Zea mays L.). Egypt. J. Appl. Sci., 15 (7): 87-102.

Soliman, F. H.S. and S. E. Sadek (1999). Combining ability of new maize inbred lines and its utilization in the Egyptian hybrid program. Bulletin Fac. Agric., Cairo Univ., 50 (1): 1-20

Sprague, G.F. and L.A. Tatum (1942). General versus specific combining ability in single crosses of corn. J. Amer. Soc. Agron., 34: 923-932.

Steel, R.G. and J. Torrie (1980). Principles and Procedures of Statistics. Mc GrawHill Book Company, New York, USA 


\section{تأثثرات القدرة الإيتلافية لبعض السلالات الجديدة من الذرة الثامية الصفراء}

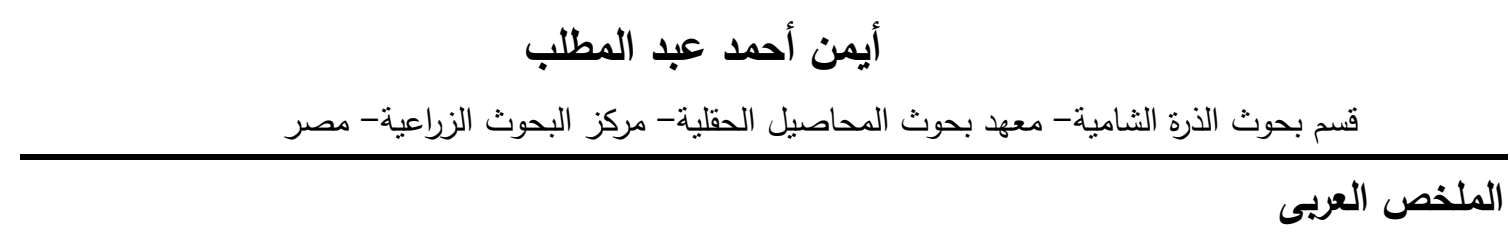

تم اجراء التهجين بين 15 سلالة مستتبطة حديثا من الذرة الثامية الصفراء مع ثلاث كثافات عبارة عن السلالة جيزة

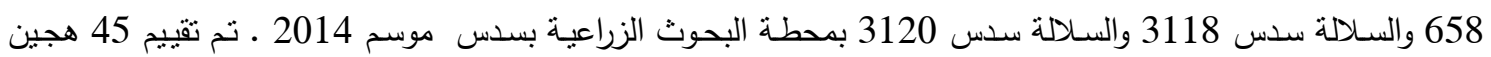
قمى مـ ثـلاث هجن مقارنـة وهما هجين فردى 162 والهجين الفردى 168 والهجين الفردى 176 فى محطتى البحوث الزراعية بالجميزة وسدس فى الموسم الزراعى 2015 ـ نم أخذ القراءات على صفات عدد الأيام من الزراعة حتى ظهور 50

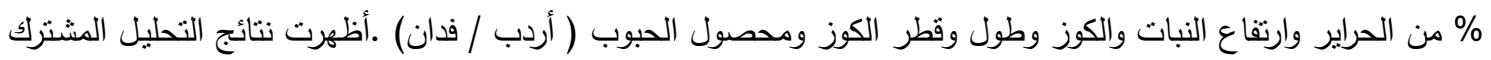

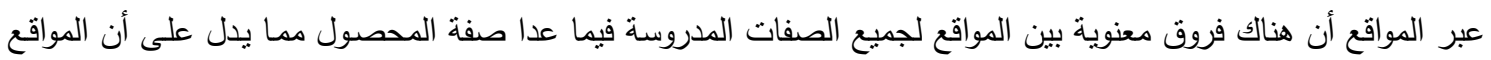
مختلفة فى الظروف البيئية. أظهر التحليل المشترك عبر المواقع اختلافات معنوية ناتجه من الهجن والسلالات والكثافات لكل الصفات تحت الدراسة ـ كما أظهر نباين تفاعل السلالات مع الكثافات اختلافات معنوية لصفات ارتفاع النبات والكوز

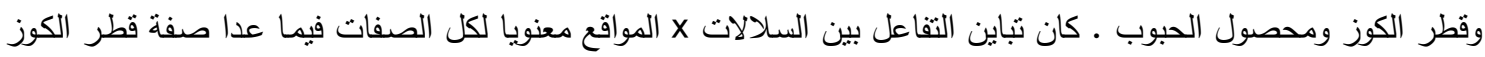

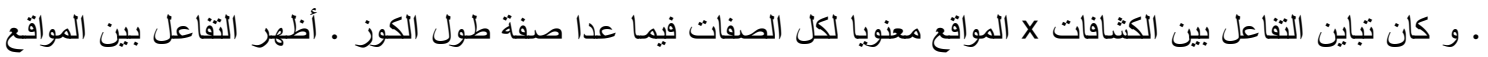

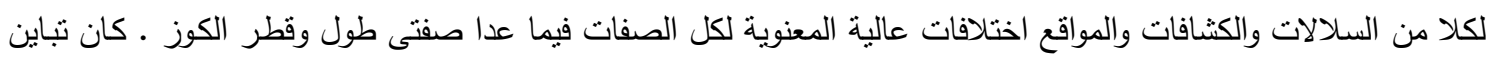
القدرة العامة على التالف اكبر من تباين القدرة الخاصة على التالف لصفة عدد الأيام من الزراعة حتى ظهور 50\% من الكن

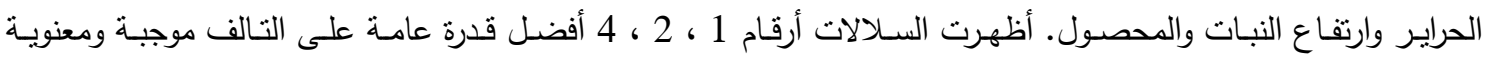

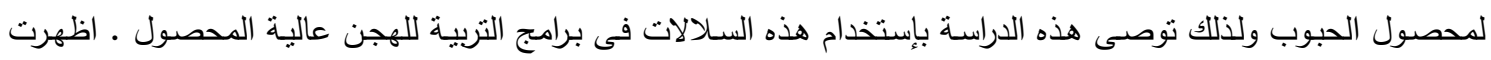

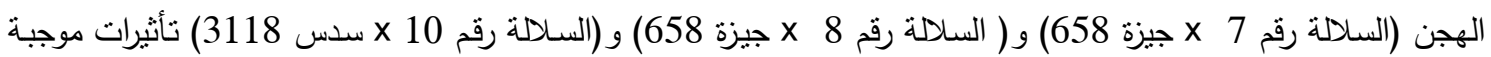
ومعنوية للقدرة الخاصة على التالف مما يمكن من استخدام هذة الهجن مباشرة كهجن تجارية بعد اجتيازها مراحل التقييم التالية ، أو يمكن استخدامها فى برامج التربية الخاصة بتحسين صفة عنة المحصول. 